\title{
The Impact of Interactive, Computerized Educational Modules on Preclinical Medical Education
}

\author{
Benjamin S. Bryner, ${ }^{1}$ Daniel Saddawi-Konefka, ${ }^{1}$ Thomas R. Gest ${ }^{2 *}$ \\ ${ }^{1}$ University of Michigan Medical School, Ann Arbor, Michigan \\ ${ }^{2}$ Division of Anatomical Sciences, University of Michigan Medical School, Ann Arbor, Michigan
}

Interactive computerized modules have been linked to improved retention of material in clinical medicine. This study examined the effects of a new series of interactive learning modules for preclinical medical education, specifically in the areas of quiz performance, perceived difficulty of concepts, study time, and perceived stress level. We randomly allocated 102 medical student volunteers into control and experimental groups. All participants studied selected anatomical and physiologic concepts using existing material (lecture notes, textbooks, etc.), while those in the experimental groups used the new interactive modules as well. All participants completed a quiz to test their knowledge of the assigned concepts and a survey to assess their subjective experiences in studying with the modules. We found a trend toward higher quiz scores in the experimental group relative to the control group, though it did not reach statistical significance $(P=0.31)$. Perceived concept difficulty was significantly reduced among those who studied with the modules $(P<0.001)$, and the number of hours spent studying the concepts was significantly increased $(P=0.028)$. Of those who used the modules, $83 \%$ rated them as "very helpful" or "extremely helpful." No significant differences existed between participants' reported stress levels during the course of the study $(P=0.44)$. Our data suggest that medical students may learn more effectively and feel less intimidated by difficult concepts when interactive modules supplement traditional instruction. Anat Sci Ed 1:247251, 2008. @ 2008 American Association of Anatomists.

Key words: undergraduate medical education; anatomical sciences/medical education; education, gross anatomy; peripheral nervous system; computers in anatomical education; interactive computer graphics

\section{INTRODUCTION}

Many studies have found computer-based interactive modules to be effective in teaching a broad range of topics encountered during the clinical phase of medical education. Residents in their first clinical year who used computerized lessons on principles of evidence-based medicine had equivalent test performance to those attending traditional lectures (Davis et al., 2007), and a series of modules on nutrition were asso-

${ }^{*}$ Correspondence to: Dr. Thomas R. Gest, Division of Anatomical Sciences, University of Michigan Medical School, 3742 Med Sci II, Ann Arbor, MI 48109. E-mail: gest@umich.edu

Received 1 September 2008; Revised 9 October 2008; Accepted 14 October 2008.

Published online 3 December 2008 in Wiley InterScience (www. interscience.wiley.com). DOI 10.1002/ase. 55

(C) 2008 American Association of Anatomists ciated with significantly higher quiz scores among pediatrics residents (Roche et al., 2007). Students who used a module on treating glomerulonephritis (a topic which they previously described as difficult) reported becoming significantly more comfortable with the topic (Velan et al., 2002).

Interactive modules are also gaining popularity at the clinical level; one study revealed that interactive modules were preferred over text, lectures, videos, or animal labs for teaching laparoscopic techniques (Ramshaw et al., 2001). Multiple web-based modules have been developed to address the concepts in radiology, ranging from introductory radiology for clinical-level medical students to interpreting the results of a highly specialized imaging modality for subspecialty radiologists (Shaffer and Small, 2004; Dikshit et al., 2005; Hassan et al., 2007). Online interactive continuing medical education (CME) for practicing physicians has become common and was found to have potential for "sustained gains in knowledge that are comparable or superior to those realized from effective live CME activities" (Fordis et al., 2005). Several medical schools have collaborated on the International Vir- 
tual Medical School (IVIMEDS), which contains hundreds of interactive "reusable learning objects" to integrate aspects of preclinical and clinical education into patient cases, and aims to eventually become a complete medical school curriculum (Harden and Hart, 2002; IVIMEDS, 2008).

Despite the empirical success of interactive computerized learning in clinical education and the growing popularity of modules for medical education in general, relatively few studies focus on quantifying the effect of interactive modules when applied to basic science concepts taught in the preclinical years of medical school. Medical students in their preclinical years are in a unique position to take advantage of computerized, interactive, self-paced instruction because of their varying schedules and increasing comfort with technology. Anatomy in particular is well suited to an interactive teaching approach because of its challenging and visually complex nature. While many tools exist for presenting anatomical information in a computerized environment, the element of interactivity is often missing. This report presents the observed impact of newly developed interactive, computerized modules on several areas of preclinical medical education, including anatomical education.

\section{METHODS}

\section{Module Development}

We asked the faculty who were involved in both designing and teaching the preclinical curriculum at our institution to suggest concepts which are typically difficult for students to master. They identified the concepts based on their teaching experience and on historic measures of student performance on examinations. Following their suggestions, we produced interactive modules on the following anatomical topics:

- Autonomic nervous system

- Lobes of the cerebral cortex

- Cranial nerves

as well as the following non-anatomical topics:

- Bayes' theorem

- The complement system

- Oxygen transport

In developing the modules, we adhered to Park and Hannafin's guidelines for maximizing the usability and benefit of interactive content (Park and Hannafin, 1993). For example, in a module on the Autonomic Nervous System (ANS), we included options for nonlinear navigation (e.g., allowing the user to skip between chapters or to change the display such that it shows only the functional division of the ANS that they are focusing on). We also offered multiple avenues for self-evaluation in the modules (e.g., games dealing with neurotransmitters at different synapses, the names of different groups of nerves, and neural pathways).

The development of each module followed a methodical four-step process, summarized in Figure 1 and detailed as follows:

1. Scope and content: We defined the scope of the material to be covered in a given module. We then performed an in-depth review of the relevant material from various sour- ces to ensure accurate and up-to-date content. This allowed us to further refine the scope of our project. By keeping our defined scope central throughout the development process, we avoided incorporating extraneous information or omitting critical concepts.

2. Design: Next, we logically arranged the content we wished to cover to make a storyboard for the module. The storyboard consisted of discrete chapters as well as the script of the narration for each chapter.

3. Production: We then produced the vector-based artwork and animation sequences that comprised the bulk of the modules in Flash, programmed the interactive features (e.g., self-assessment features and nonlinear navigation), and recorded and synchronized the audio content.

4. Review: Finally, we thoroughly tested each module to ensure seamless technical performance, and the faculty reviewed the modules to make certain that there were no content errors.

The modules were all produced in an interactive framework of authors' own design created with Macromedia Flash MX 2004 (Macromedia, San Francisco, CA [since bought by Adobe Systems, San Jose, CA]). Each module is divided into chapters and includes animation, narration, interactive illustration of principles, and opportunities for self-assessment (see Fig. 2). We relied on copyrighted images for the Cranial Nerves and Lobes of the Cerebral Cortex modules (which meant they could only be used within our institution), but we generated our own images for the four other modules to ensure wider availability. The four publicly available modules can be viewed online at the Medical Gross Anatomy Learning Resources of the University of Michigan Medical School (M1 Supplemental Modules, 2008).

\section{Module Evaluation}

The study was open to all students at our institution between their first and second years of medical school, as well as students in a "pre-matriculation" summer program (an optional preparatory session immediately before the first year of medical school). To maximize the total number of modules tested without overburdening participants, we divided study participants into three groups. The first group consisted of students between their first and second years of medical school; this group was assigned to study Oxygen Transport, Cortical Lobes, and Cranial Nerves. The second group, also consisting of students between their first and second years of medical school, studied Bayes' Theorem and the Complement System. The third group, consisting of students in the prematriculation program, studied the ANS and Oxygen Transport.

Each of these three groups was divided evenly into control and experimental groups using a computer-based random assignment algorithm. Control group participants studied their assigned concepts using only existing materials (lecture slides, notes, textbooks, review books, etc.). Experimental group participants were allowed to use the same materials, as well as the relevant modules. Since all participants were busy with research or classes at the time of the study, they were given a period of 5 weeks to study the assigned topics and take the online survey and quiz. This model of flexible testtaking is similar to the one employed during the academic year at our institution.

We asked the same faculty members who had suggested topics to develop quiz questions in order to evaluate appro- 


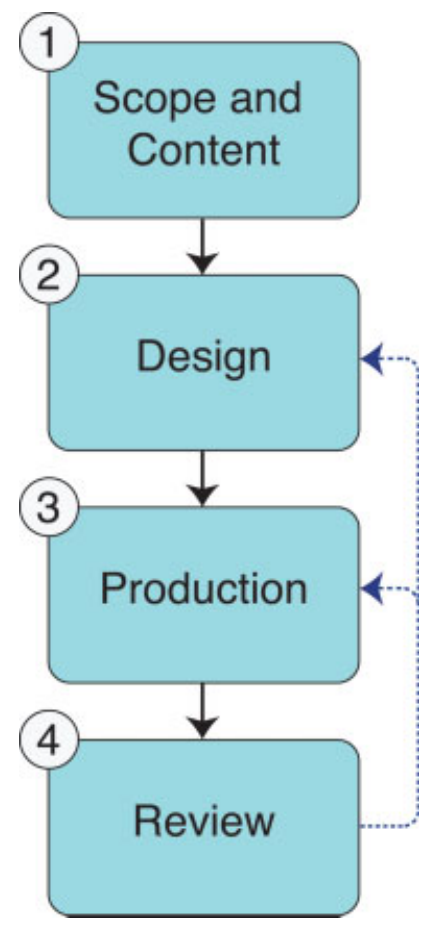

\section{Figure 1.}

A four-step approach to developing interactive computerized modules.

priate mastery of content. To avoid biasing the content of the quizzes, we obtained the questions from the faculty before they reviewed the modules for accuracy. The questions were written in the same format as those used during preclinical medical student examinations. Nine to 12 multiple-choice questions were collected per topic to create the quizzes, and the quizzes were administered through the same web-based system used for preclinical examinations. Participants were only asked questions relevant to their assigned topics. Quizzes were "closed book" (i.e., participants were not allowed to consult any materials while taking the quiz). Individual results were not made available to anyone besides the investigators of this study.

After taking the quiz, participants completed a survey to assess the modules' effect on studying time, perceived level of difficulty of the concepts tested, and perceived stress level while studying and taking the quiz. Participants had been asked to track their study time (to the nearest $6 \mathrm{~min}$ ). A seven-point scale was used to assess participants' perceived difficulty of the topics being tested ( 1 being "extremely easy" and 7 being "extremely difficult"). With regard to perceived stress level, participants were asked to describe the level of stress they felt during the study on the following scale: "no stress," "very little stress," "little stress," "little to moderate stress," "moderate stress," "moderate to high stress," "high stress," "very high stress."

We also asked participants in the experimental group to rate how helpful the modules were ("not helpful," "somewhat helpful," "very helpful," or "extremely helpful”) as well as to provide any comments on the modules. After the control group participants took their quizzes, they were given access

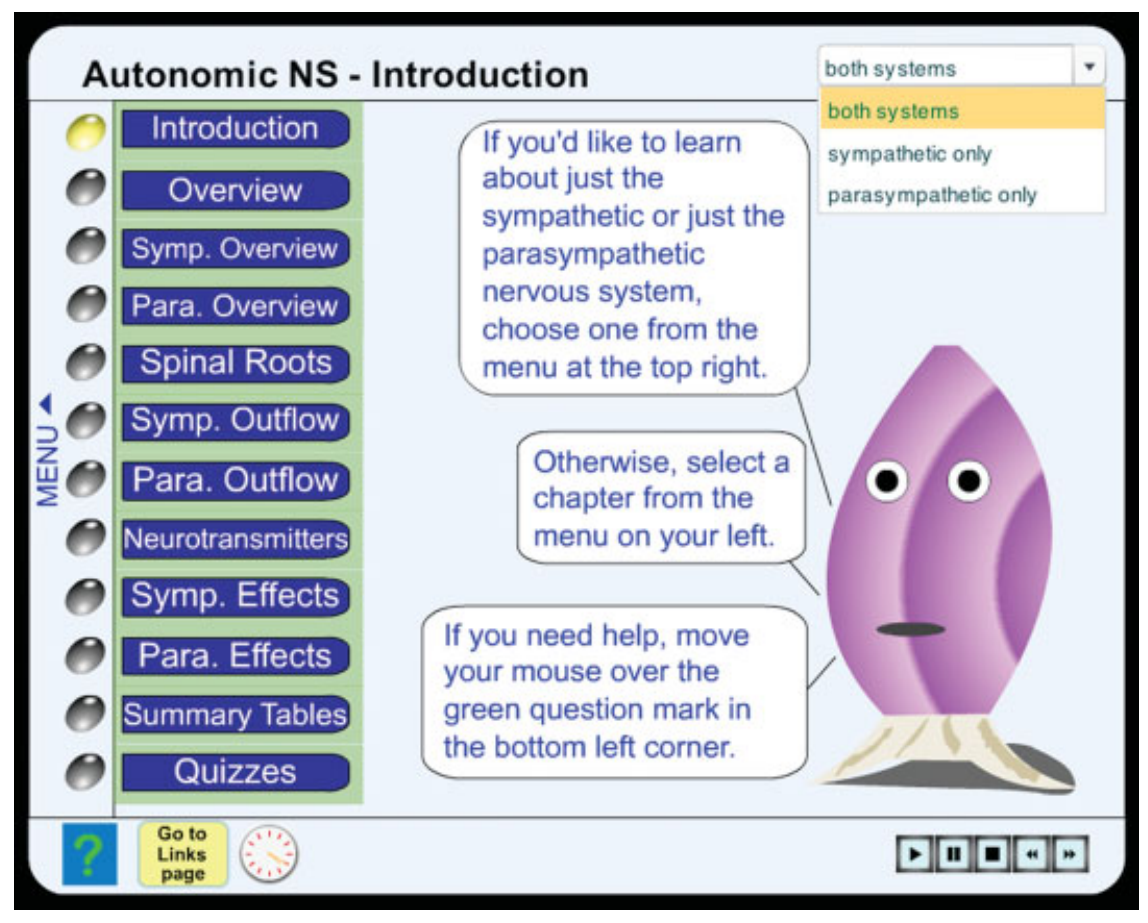

Figure 2.

Introductory screen for the Autonomic Nervous System (ANS) module. The module allows the user to explore the divisions of the ANS separately or together, and it gives opportunities for self-evaluation through games. 


\begin{tabular}{|c|c|c|c|c|}
\hline & Experimental group ${ }^{a}$ & Control group ${ }^{b}$ & $T$ statistic & $P$ value \\
\hline$n$ (randomized to groups) & 51 & 53 & & \\
\hline$n$ (actually completed quiz) & 42 & 34 & & \\
\hline Quiz score & $78.8 \% \pm 16.4 \%$ & $74.7 \% \pm 18.4 \%$ & 1.02 & 0.31 \\
\hline Study time (hours per topic) & $1.13 \pm 0.90$ & $0.89 \pm 0.59$ & 2.21 & $0.028^{\mathrm{c}}$ \\
\hline Stress (0-7 scale) & $3.74 \pm 1.19$ & $3.51 \pm 1.49$ & 0.78 & 0.44 \\
\hline Perceived difficulty of topic (1-7 scale) & $3.05 \pm 1.31$ & $4.10 \pm 1.48$ & 5.28 & $<0.001^{\mathrm{c}}$ \\
\hline
\end{tabular}

${ }^{\mathrm{a} E x p e r i m e n t a l ~ g r o u p ~ w a s ~ a l l o w e d ~ t o ~ u s e ~ r e l e v a n t ~ m o d u l e s . ~}$

${ }^{\mathrm{b}}$ Control group did not use modules.

${ }^{\mathrm{c}}$ Statistically significant results $(\alpha=0.05)$.

to the interactive modules and asked to share their opinions on them as well.

Quiz scores and other statistics were analyzed with independent-sample $t$ tests within SPSS version 15 (SPSS, Chicago, IL). The study was granted an exemption from institutional review board oversight under the provision for researching educational methods.

\section{RESULTS}

Quiz scores and other statistics are summarized in Table 1. The participants in the experimental group had a higher mean quiz score for every concept studied (79\% vs. $75 \%$ overall), though no statistical significance was present overall $(t=1.02, P=0.31)$.

The perceived level of difficulty of the tested concepts was significantly lower in the experimental group (3.0 vs. 4.1 on a scale of 1 to $7 ; t=5.28, P<0.001)$. Study time was significantly higher in the experimental group $(1.1 \mathrm{hr}$ vs. $0.89 \mathrm{hr} ; t$ $=2.21, P=0.028)$. No significant differences were observed for perceived stress levels between the control and experimental groups $(3.7$ vs. 3.5 on a scale of 0 to $7 ; t=0.783, P=$ 0.44). Dropout rates were significantly higher in the control group than in the experimental group $\left(27 \%\right.$ vs. $18 \% ; \chi^{2}=$ $4.38, P=0.036)$. Of the 50 participants who had access to the modules, zero participants described the modules as "not helpful," 7 (17\%) described the modules as "somewhat helpful," $22(52 \%)$ called them "very helpful," and $13(31 \%)$ called them "extremely helpful."

In their comments, students most commonly expressed appreciation for the following aspects of the modules: conciseness of presentation, self-explanatory nature, multisensorial learning modalities, utility of links to other resources with more details or with different approaches, and ready availability of the modules anywhere with Internet access.

\section{DISCUSSION}

Although interactive resources for clinical education are widely used and evaluated, evidence of the effectiveness of these tools in the setting of preclinical education is less abundant. Given the increased comfort the new generations of physicians have with technology and the increased focus that has been placed on technological supplements in preclinical education, this issue deserves investigation. Assessing different perceptual modalities may be important in future studies as well. Overall, our experience indicates that interactive computerized modules are indeed a useful supplement to help students understand and master traditionally difficult concepts in preclinical medical education.

Our study has some limitations. First, high dropout rates were observed across both the control and experimental groups with significantly more dropping out of the control group. Retrospective follow-up with these individuals revealed no qualitative differences in the type of individual dropping out. Second, in following our four-step approach to develop interactive modules, the form and presentation style of the concepts was unavoidably altered. Thus, our study examined not simply the effects of computerized modules in isolation, but also the effects of computerized modules as developed through our systematic four-step approach. Thus, in efforts to recreate the exact results of this study, our structured approach to interactive module development should be followed.

Our study also has several strengths. We involved core faculty in identifying topics and tested the modules on actual medical students to make our results directly applicable to premedical education. Additionally, by specifically selecting the topics which the faculty considers difficult for students, we believe that our results will be generalizable to a wider range of topics.

Based on our investigation, we are able to draw several key conclusions. First, if interactive modules are presented concomitantly with traditional (i.e., noninteractive) material, quiz performance is at least as high as it is without them. Higher-powered studies may be able to find significance in the trend toward higher scores that we observed. Second, when interactive modules supplement traditional curricula, students will spend more time studying the concepts. While this may be due to an overabundance of learning resources, participants' comments lead us to believe that it is actually due, at least in part, to an increased desire to devote time to 
learning these concepts. Third, our results showed no decrease in participants' perceived stress levels while using the modules. We had anticipated a decrease in stress levels. Based on some user comments, we believe that the higher reported stress level reflects in part frustration about a mathematical error in one of the modules (which they were not allowed to discuss with us until after the study to avoid bias). Also, the fact that this study was not conducted under medical school conditions (i.e., our participants' scores were not reflected in medical school grades, and this study was not a major demand on their time) makes the stress levels difficult to interpret. It is also possible that the higher dropout rate in the control group was due to stress-related factors that we were not able to capture in our survey. Our last key conclusion, that is, perceived concept difficulty was significantly lowered in those who studied with the modules, is especially noteworthy given that difficult concepts were selected as topics for our modules. This underscores the potential for interactive modules to be applied to topics that traditionally require significant effort to teach.

Our experience in developing the modules allows us to make several recommendations to educators who are considering creating their own. On a technical note, using Flash, a vector-based program that allows incorporation of audio, video, and interactive elements, allowed for wide and rapid distribution of the modules. Anatomical information was especially adaptable to the interactive format, as there are multiple ways to visually engage students when teaching anatomy. Although Flash contains many powerful tools for developing interactive modules, its steep learning curve demands a significant time investment up front. The authors had some programming experience, but the process of learning Flash and constructing the modules did take a significant amount of time. This necessitates careful planning and realistic goals. Educators should recognize these technical issues as potential challenges. Taking all of these factors into account, the level of investment required to create similar modules will depend both on the platform selected and on the level of developer's previous level of technical expertise. With regard to the development process, the four-step approach presented in the "Methods" section of this report can serve as a guide for interested educators.

In sum, interactive modules decrease perceived concept difficulty, have the potential to increase test performance, and likely increase students' desire to study material. The results outlined in this report underscore the need for further exploration of the potential of interactive modules in preclinical education.

\section{NOTES ON CONTRIBUTORS}

BENJAMIN S. BRYNER, M.S., is a student at the University of Michigan Medical School in the Class of 2009, and a trainee in the Multidisciplinary Clinical Researchers in Train- ing Program at the University of Michigan School of Public Health in Ann Arbor, Michigan.

DANIEL SADDAWI-KONEFKA, B.S., is a dual degree student at the University of Michigan Medical School in Ann Arbor, Michigan, and the University of Michigan Ross School of Business in the Class of 2009.

THOMAS R. GEST, Ph.D., is an associate professor of anatomical sciences and the director of Medical Gross Anatomy in the Division of Anatomical Sciences at the University of Michigan Medical School, Ann Arbor, Michigan. He is a director of the Anatomical Donations Program at the University of Michigan and has a longstanding interest in researching anatomical and general medical education.

\section{ACKNOWLEDGMENTS}

The authors thank Dr. Louis D'Alecy, Dr. Joseph Fantone, Dr. Thomas Gelehrter, Dr. Raj Mangrulkar, Dr. Sarah Newman, and Dr. Robert Paine for assisting with content and for providing quiz questions for the evaluation of the modules; Chris Chapman, Jason Engling, and John Westfall for technical assistance; and Brenda Chism and Cynthia Sharp for administering the quizzes.

\section{LITERATURE CITED}

Davis J, Chryssafidou E, Zamora J, Davies D, Khan K, Coomarasamy A. 2007. Computer-based teaching is as good as face to face lecture-based teaching of evidence based medicine: A randomised controlled trial. BMC Med Educ 7:23.

Dikshit A, Wu D, Wu C, Zhao W. 2005. An online interactive simulation system for medical imaging education. Comput Med Imaging Graph 29:395-404.

Fordis M, King JE, Ballantyne CM, Jones PH, Schneider KH, Spann SJ, Greenberg SB, Greisinger AJ. 2005. Comparison of the instructional efficacy of Internet-based CME with live interactive CME workshops: A randomized controlled trial. J Am Med Assoc 294:1043-1051.

Harden RM, Hart IR. 2002. An international virtual medical school (IVIMEDS): The future for medical education? Med Teach 24:261-267.

Hassan BA, Jacobs R, Scarfe WC, Al-Rawi WT. 2007. A web-based instruction module for interpretation of craniofacial cone beam CT anatomy. Dentomaxillofac Radiol 36:348-355.

IVIMEDS, International Virtual Medical School. 2008. Dundee, Scotland, UK. URL: http://www.ivimeds.org [accessed 19 September 2008].

M1 Supplemental Modules. 2008. Medical Gross Anatomy Learning Resources. Ann Arbor, MI: University of Michigan Medical School. URL: http://anatomy. med.umich.edu/bnb [accessed 19 September 2008].

Park I, Hannafin M. 1993. Empirically-based guidelines for the design of interactive multimedia. Educ Technol Res Dev 41:63-85.

Ramshaw BJ, Young D, Garcha I, Shuler F, Wilson R, White JG, Duncan T, Mason E. 2001. The role of multimedia interactive programs in training for laparoscopic procedures. Surg Endosc 15:21-27.

Roche PL, Ciccarelli MR, Gupta SK, Hayes BM, Molleston JP. 2007. Multischool collaboration to develop and test nutrition computer modules for pediatric residents. J Am Diet Assoc 107:1586-1589.

Shaffer K, Small JE. 2004. Blended learning in medical education: Use of an integrated approach with web-based small group modules and didactic instruction for teaching radiologic anatomy. Acad Radiol 11:1059-1070.

Velan GM, Killen MT, Dziegielewski M, Kumar RK. 2002. Development and evaluation of a computer-assisted learning module on glomerulonephritis for medical students. Med Teach 24:412-416. 